\title{
Lung function and exercise capacity in thalassaemia major
}

\author{
C. Cracowski*, B. Wuyam*, V. Klein**, P. Lévy*
}

Lung function and exercise capacity in thalassaemia major. C. Cracowski, B. Wuyam, V. Klein, P. Lévy. @)ERS Journals Ltd 1998.

ABSTRACT: Lung function abnormalities in thalassaemia major are various and complex; however, patients still die from cardiac lesions. This study aimed to investigate pulmonary and cardiac involvement at an early stage in thalassaemic patients and study their respective implications at rest and during exercise.

Ten patients (five adults and five children) with thalassaemia major were investigated by echocardiography, lung function and exercise testing a few days after transfusion. All have had regular transfusions and chelation with deferoxamine and none had chronic pulmonary disease symptoms.

Minor lung function abnormalities were found: two patients had moderate obstructive syndrome and two had a decreased carbon monoxide transfer factor. Hypoxaemia was never found at rest and no desaturation was observed at the end of exercise. Echocardiographic abnormalities were also moderate. Peak oxygen consumption $\left(V^{\prime} \mathrm{O}_{2}\right)$ was decreased in three adults and was lower in adults than children (means $27.7 \pm 4.6$ and $41.1 \pm 4.8 \mathrm{~mL} \cdot \mathrm{kg}^{-1} \cdot \mathrm{min}^{-1}$ respectively). The $V^{\prime} \mathrm{o}_{2} /$ cardiac frequency slope was lower in adults than children $\left(0.25 \pm 0.06\right.$ versus $\left.0.42 \pm 0.10 \mathrm{~mL} \cdot \mathrm{kg}^{-1} \cdot \mathrm{min}^{-2}\right)$, whereas end-exercise breathing reserve was $>40 \%$ maximal voluntary ventilation for all patients.

In conclusion, none of the patients had ventilatory limitations but older patients had cardiac limitations assessed by the relationship between oxygen consumption and cardiac frequency. Exercise testing may detect cardiac impairment in thalassaemia major earlier than investigations at rest.

Eur Respir J 1998; 12: 1130-1136.
*Cardiopulmonary Laboratory and **Dept of Paediatrics, Grenoble Hospital, France.

Correspondence: B. Wuyam

Laboratoire EFCR

Hôpital A. Michalon

BP 217 X- 38043 Grenoble-Cédex 09

France

Fax: 33476765617

Keywords: Cardiac limitation exercise testing

lung function

Received: December 191997

Accepted after revision July 181998
Thalassaemia major is characterized by a congenital abnormality of globulin chain synthesis, ineffective erythropoiesis and severe anaemia. Treatment is generally based on regular transfusions in addition to iron chelation therapy (due to iron overload). Such treatment has been shown to be effective in reducing overall mortality, which is most frequently due to cardiac complications [1,2]. The improved prognosis allows new clinical features to emerge, in particular lung function impairment.

Initial studies regarding lung function, however, were quite contradictory. COOPER et al. [3] demonstrated a restrictive pattern in some patients that tended to correlate with age and hence the duration of the disease. Conversely, in the same year, KeENS et al. [4] described abnormalities in lung function test results suggestive of a small airway dysfunction which, they postulated, could be due to iron deposits in peripheral airways. Since then, studies have revealed either an obstructive [5] or more frequently a restrictive pattern [6-9]. In addition, several authors have suggested the possibility of pulmonary vascular involvement by microthrombosis. This was initially suggested on histological findings after autopsy [10]. Pulmonary hypertension was later detected in some patients by echocardiography [7] and right heart catheterization [11]. Finally, the pulmonary consequences of abnormal cardiac function may interfere with lung involvement by the disease and/or its treatment (iron overload) and this may account for at least some of the abnormalities of lung function test results.
Therefore, this study was undertaken to assess lung function and simultaneously conduct echocardiography and cardiopulmonary exercise tests in thalassaemic patients. The aims were 1) to detect potential pulmonary hypertension and/or other forms of lung involvement at an early stage and 2) to investigate whether exercise tolerance would be predominantly affected by cardiac or pulmonary abnormalities.

\section{Methods}

\section{Patients}

Eleven subjects were recruited from the author's institution between December 1995 and March 1996: six children and adolescents (aged 6-18 yrs), followed by the Paediatric Department, and five adults (aged 31-35 yrs), followed by the outpatient clinic of the Haematology Department. One patient was excluded from the analysis owing to a combination of factors affecting respiratory function and not directly related to thalassaemia present in this subject, i.e. obesity $(1.56 \mathrm{~m}, 66 \mathrm{~kg})$, abdominal parietal defect after surgery and kyphoscoliosis. The excluded patient also suffered from occasional symptoms of allergic asthma and received inhaled bronchodilator and anti-inflammatory drug therapy. None of the other subjects had symptoms compatible with chronic lung disease. All patients or their parents gave their informed consent to participate in the 
study. A diagnosis of major $\beta$-thalassaemia was made in the first year of life for eight out of the 10 patients, and at 3 and 4 yrs of age for the other two patients. All patients were treated by regular transfusions of erythrocytes (mean volume $545 \mathrm{~mL}$, range $300-750 \mathrm{~mL}$ ) every 3-7 weeks. Transfusions began at the time of diagnosis for all patients and were maintained throughout the evolution of the disease. In addition, regular iron chelation was administered using deferoxamine (DFO) injection either intravenously or sub-cutaneously (mean dose of $1.3 \mathrm{mg} \cdot \mathrm{kg}^{-1} \cdot \mathrm{month}^{-1}$, range 0.6-2.6). Chelation therapy was started $0-3$ yrs after the beginning of transfusions for the five children and 4-8 yrs for the five adults. Compliance with therapy was good. Treatment with DFO was not permanently maintained, how-ever, especially in adults where culminated time without chelation therapy reached 5-12 yrs (versus 0.3 yrs in children). The duration for which serum ferritin was elevated (arbitrarily, greater than three times the normal value, i.e. $1,000 \mu \mathrm{g} \cdot \mathrm{L}^{-1}$ ) was evaluated in each subject and was 2.5-11 yrs for children and 17-35 yrs for adults, despite chelation therapy. All subjects were nonsmokers. Two patients (Nos. 9 and 10) had had acute respiratory symptoms at-tributed to DFO toxicity which proved clinically rever-sible after interruption of the treatment. Two of the five children and all adults suffered from hepatosiderosis of various intensity, two adults suffered from glucose intolerance and all adults suffered from endocrine insufficiency (four with hypoparathyroidism, three with sex hormone fail-ure, one with hypothyreosis and one with growth hormone insufficiency) and received hormone-replacement therapy. All adults and children had an active and socially well-in-tegrated lifestyle.

\section{Lung function testing}

Lung function tests were performed 1-4 days after transfusion. For adults, lung volumes and forced ventilator flows were determined with a computerized water-seal spirometer (Volugraph® VG 2000; Volugraph, Mijnhardt, The Netherlands). In children and adolescents, a calibrated heated Fleisch (No. 2 and 3) pneumotachograph was used for spirometry (Eolys $®$; Lyon, France). Functional residual capacity (FRC) was determined by helium dilution. For children, the helium circuit volume was reduced $(<4 \mathrm{~L})$ and two reproducible measures of FRC $( \pm 10 \%)$ were obtained in all children. Reference values were the 1983 European Community for Coal and Steel reference values for adult Caucasians [12] and those of ZapLeTAL et al. [13] for children and adolescents.

The carbon monoxide transfer factor $(T \mathrm{~L}, \mathrm{CO})$ and coefficient $(\mathrm{KCO})$ were measured by the single breath-holding method using a calibrated infrared CO analyser (PK Morgan, Gillingham, Kent, UK). TL,CO was expressed at a standard haemoglobin concentration $(\mathrm{Hb})$ of $146 \mathrm{~g} \cdot \mathrm{L}^{-1}$ for males and $136 \mathrm{~g} \cdot \mathrm{L}^{-1}$ for females. Reference values were those of Coтes et al. [14].

\section{Blood gas analyses}

Arterial blood gas were sampled at rest in a supine posture and analysed (Corning 178; Ciba-Corning, Medfield, MA, USA; ABL 500; Radiometer, Copenhagen, Denmark). Arterial oxygen saturation $\left(\mathrm{Sa}_{\mathrm{a}} \mathrm{O}_{2}\right)$ and $\mathrm{Hb}$ concen- tration were measured with an haemoximeter (OMS 3; Radiomet-er) calibrated for the presence of foetal haemoglobin $(\mathrm{HbF})$.

\section{Exercise testing}

Exercise studies were performed within 3-5 days after transfusion. Subjects exercised on a mechanically braked cycle ergometer (Ergedic 818, Monark®; Varberg, Sweden) using a $15 \mathrm{~W} \cdot \mathrm{min}^{-1}$ incremental protocol until exhaustion. Maximal exercise was determined using standard criteria [15]. In young children $(<10 \mathrm{yrs})$, treadmill exercise was used with a constant treadmill speed of $5 \mathrm{~km} \cdot \mathrm{h}^{-1}$ and progressive increase in slope of $2.5 \% \cdot \mathrm{min}^{-1}$. Expired volumes were measured using a Fleisch 3 pneumotachograph. Oxygen consumption $\left(V^{\prime} \mathrm{O}_{2}\right)$ and carbon dioxide production $\left(V^{\prime} \mathrm{CO}_{2}\right)$ were derived from $30 \mathrm{~s}$ measurements of $\mathrm{O}_{2}$ (zirconium $\mathrm{O}_{2}$ analyser) and $\mathrm{CO}_{2}$ (infrared $\mathrm{CO}_{2}$ analyser) fractional concentrations in a $7 \mathrm{~L}$ mixing chamber (Metasys TM®; Brainware, La Salette du Var, France). The peak $V^{\prime} \mathrm{O}_{2}$ measurements were expressed as per cent of predicted normal values for sedentary adults and children [15]. End-exercise ventilation was expressed as a percentage of maximal voluntary ventilation (MVV) calculated by the formula: $\mathrm{MVV}=35 \times$ forced expiratory volume in one second (FEV1) [15]. The ventilatory threshold (VT) was determined using the ventilatory equivalent method: minimal $V^{\prime} \mathrm{E}$ (ventilation) $/ V^{\prime} \mathrm{O}_{2}$ preceding the increase in $V^{\prime} \mathrm{E} / V^{\prime} \mathrm{CO}_{2}$ [15] and the change in the slope of the $V^{\prime} \mathrm{CO}_{2} / V^{\prime} \mathrm{O}_{2}$ relationship. A good agreement between the two methods was observed in all subjects. $\mathrm{Sa}_{\mathrm{a}} \mathrm{O}_{2}$ was monitored using an earlobe pulse oximeter (Satlite trans®; Datex, Helsinki, Finland). At the end of exercise (1 min after cessation), lactataemia was determined by micropuncture recovery of $20 \mu \mathrm{L}$ of capillary blood. Determination was performed by enzymatic dosage (Microzym L®; SGI, Toulouse, France).

\section{Echocardiography}

Echocardiography was performed in all subjects 1-28 days after transfusion. A unique operator performed the echocardiography in each group of adults (Hewlett Packard 1500 and/or 2500 echocardiograph with 2.0 and 2.5 $\mathrm{MHz}$ transducers) and children (Kontron Sigma 44 with a $5 \mathrm{MHz}$ transducer). M-Mode recordings of left ventricular (LV) dimensions at end-diastole and end-systole and right ventricular (RV) diameter at end-diastole were obtained from the parasternal view. The left ventricle shortening fraction was then calculated from the M-mode tracings [16]. The thickness of the interventricular septum (IVS) and posterior wall (LVPW) were also measured.

The apical four-chamber view was analysed to study RV systolic function. The two-dimensional RV shortening fraction was calculated from the end-diastolic and systolic area. Doppler studies were performed with pulsed and continuous-wave Doppler probes. Systolic pulmonary artery pressures (sPAP) were calculated from the right atriumventricle pressure gradient $(\mathrm{RA}-\mathrm{RV}$ ) using the tricuspid insufficiency flow velocity by the following formula: sPAP $=\mathrm{RA}-\mathrm{RVg}+10 \mathrm{mmHg}$. Cardiac output was calculated from the aortic flow, aortic diameter and cardiac frequency $(f \mathrm{C})$. As proposed by Feignebaum [16], left and right diastolic 
diameters were expressed as values normalized for differences in body surface area $\left(\mathrm{cm} \cdot \mathrm{m}^{-2}\right)$.

Statistical analysis

Group data were expressed as mean \pm sD. Between-group comparison (adults versus children) were made with a nonparametric Mann-Whitney test. Correlation analysis was performed using a nonparametric Spearman's test. Results were considered as significant for $\mathrm{p}$-values $<0.05$.

\section{Results}

\section{Biological data}

The mean $\mathrm{Hb}$ count after transfusion was $110 \pm 22 \mathrm{~g} \cdot \mathrm{L}^{-1}$. Three patients (Nos. 1, 5 and 7) had a $[\mathrm{Hb}]<100 \mathrm{~g} \cdot \mathrm{L}^{-1}$ even after transfusion. The mean pretransfusional level of $\mathrm{Hb}$ over the preceding months was $81 \pm 7 \mathrm{~g} \cdot \mathrm{L}^{-1}$. The serum ferritin level was elevated at the time of the study: mean $=1633 \pm 1340 \mu \mathrm{g} \cdot \mathrm{L}^{-1}$ range $400-4,600 \mu \mathrm{g} \cdot \mathrm{L}^{-1}$ (normal 5$\left.350 \mu \mathrm{g} \cdot \mathrm{L}^{-1}\right)$.

\section{Lung function testing and blood gases at rest}

Lung function was minimally affected in this group of patients. Two patients (Nos. 3 and 7) displayed airway obstruction, defined by a decrease in FEV1/vital capacity (VC) $\partial 90 \%$ predicted (table 1). None of them had clinical symptoms of asthma. Nor did any of the patients have a restrictive defect, defined by total lung capacity (TLC) $<80 \%$ pred, and the mean TLC for the group was $96 \pm 8 \%$ pred. A diffusion abnormality, i.e. KCO $<80 \%$ pred was detected in two of the five adult patients (patients No. 1 and 3) but none of the children. All patients had normal blood gases at rest, in particular, none of the patients, neither adults nor children, were hypoxaemic at rest (group mean arterial oxygen tension $\left(\mathrm{Pa}, \mathrm{O}_{2}\right)$ of $\left.13.1 \pm 1.1 \mathrm{kPa}\right)$.
Mean $\mathrm{Sa}_{2} \mathrm{O}_{2}$ was lower in adults than in children: $94 \pm 1$ and $96 \pm 1 \%$, respectively $(\mathrm{p}=0.01)$.

\section{Exercise capacity}

All subjects reached a near-maximal $f \mathrm{C}$ at the end of exercise (group mean $f \mathrm{C} 180 \pm 11$ beats. $\mathrm{min}^{-1}, 91 \pm 6 \%$ of predicted maximal $f C$ (table 2$)$ ). The respiratory exchange ratio $\left(\mathrm{RER}=V^{\prime} \mathrm{CO}_{2} / V^{\prime} \mathrm{O}_{2}\right)$ at the end of exercise was high (group mean RER of $1.12 \pm 0.07$ ); this was particularly so in adults where the group mean RER was $1.20 \pm 0.06$. This suggests that maximal exercise was achieved.

A decrease in exercise capacity was observed, at least in some of the patients (Nos. 1, 3 and 4). The group mean peak $V^{\prime} \mathrm{O}_{2}$ achieved was $86 \pm 19 \%$ pred normal sedentary subjects. Subgroups of adults or children were differently affected: adults had a significantly lower peak $V^{\prime} \mathrm{O}_{2}$ (mean of $27.7 \pm 4.6 \mathrm{~mL} \cdot \mathrm{kg}^{-1} \cdot \mathrm{min}^{-1}, 76 \pm 10 \%$ pred) than children (mean of $41.1 \pm 4.8 \mathrm{~mL} \cdot \mathrm{kg}^{-1} \cdot \mathrm{min}^{-1}, 94 \pm 22 \%$ pred); ( $=0.03$ ). None of the patients had ventilatory limitation, as assessed by an end-exercise ventilation at $59.2 \pm 10.7 \%$ MVV, (normal (N) Š $40 \%$ ). Breathing reserve at the end of exercise was $>15 \mathrm{~L} \cdot \mathrm{min}^{-1}$ in all patients [15]. The group mean ventilatory threshold was $55 \pm 15 \%$ of peak $V^{\prime} \mathrm{O}_{2}$. One subject (patient No. 3) had a low ventilatory threshold at $38 \%$ peak $V^{\prime} \mathrm{O}_{2}$ pred. Mean ventilatory threshold was significantly lower in adults than in children $(48 \pm 8 \%$ versus $66 \pm 8 \%$ of peak $V^{\prime} \mathrm{O}_{2}$, respective-ly; $\mathrm{p}=0.03$ ).

$\mathrm{Sa}_{\mathrm{a}} \mathrm{O}_{2}$ was stable during exercise in all patients, with changes in $\mathrm{O}_{2}$ saturation of $<4 \%$. There was no argument in favour of an increased dead space during exercise; in particular, the $V^{\prime} \mathrm{E} / V^{\prime} \mathrm{CO}_{2}$ ratio at the ventilatory threshold was within normal limits (mean group $V^{\prime} \mathrm{E} / V^{\prime} \mathrm{CO}_{2}=30.2 \pm$ $4.0 ; \mathrm{N}=29.1 \pm 4.3$ ). Lactataemia at the end of exercise was $7.1 \pm 1.5 \mathrm{mmol} \cdot \mathrm{L}^{-1}$ in adults and $6.3 \pm 0.5 \mathrm{mmol} \cdot \mathrm{L}^{-1}$ in children. $V^{\prime} \mathrm{O}_{2}$ (normalized for differences in body weight) $/ f \mathrm{C}$ relationships in adults and children are shown in figure 1. $V^{\prime} \mathrm{O}_{2} / f \mathrm{C}$ relationships could be adequately described by linear functions in all patients (values of the regression cor-

Table 1. - Lung function data

\begin{tabular}{|c|c|c|c|c|c|c|c|c|c|c|c|c|c|c|c|c|c|c|}
\hline \multirow{2}{*}{$\begin{array}{l}\text { Patients } \\
\text { no. }\end{array}$} & \multirow{2}{*}{$\begin{array}{l}\text { Age } \\
\text { yrs }\end{array}$} & \multirow{2}{*}{$\begin{array}{l}\text { Weight } \\
\mathrm{kg}\end{array}$} & \multirow{2}{*}{$\begin{array}{l}\text { Height } \\
\mathrm{m}\end{array}$} & \multirow{2}{*}{$\begin{array}{c}\text { BSA } \\
\mathrm{m}^{2}\end{array}$} & \multicolumn{2}{|c|}{ FEV1 } & \multicolumn{2}{|c|}{$\mathrm{VC}$} & \multicolumn{2}{|c|}{ FEV1/VC } & \multicolumn{2}{|c|}{ TLC } & \multirow{2}{*}{$\begin{array}{c}\text { KCO } \\
\% \text { pred }\end{array}$} & \multirow{2}{*}{$\begin{array}{l}P \mathrm{a}, \mathrm{O}_{2} \\
\mathrm{kPa}\end{array}$} & \multirow{2}{*}{$\begin{array}{c}{\mathrm{Pa}, \mathrm{CO}_{2}}_{2} \\
\mathrm{kPa}\end{array}$} & \multirow[t]{2}{*}{$\mathrm{pH}$} & \multirow{2}{*}{$\begin{array}{c}{\mathrm{Sa}, \mathrm{O}_{2}}_{\%} \\
\%\end{array}$} & \multirow{2}{*}{$\begin{array}{l}\mathrm{Hb} \\
\mathrm{g} \cdot \mathrm{L}^{-1}\end{array}$} \\
\hline & & & & & $\mathrm{L}$ & $\%$ pred & $\mathrm{L}$ & $\%$ pred & $\%$ & $\%$ pred & $\mathrm{L}$ & $\%$ pred & & & & & & \\
\hline \multicolumn{19}{|l|}{ Adults } \\
\hline 1 & 35 & 65 & 1.78 & 1.78 & 4.02 & 97 & 5.38 & 103 & 75 & 93 & 7.10 & 99 & 72 & 13.7 & 4.9 & 7.45 & 95 & 94 \\
\hline 2 & 33 & 51 & 1.56 & 1.45 & 2.74 & 97 & 3.23 & 101 & 83 & 100 & 4.27 & 95 & 97 & & 4.1 & 7.44 & 94 & 134 \\
\hline 3 & 33 & 63 & 1.58 & 1.60 & 3.08 & 91 & 4.28 & 105 & 72 & 88 & 5.38 & 97 & 73 & 12 & 4.9 & 1 & 93 & 103 \\
\hline 4 & 31 & $T$ & .56 & 1.42 & 2.34 & 84 & 2.89 & 89 & 81 & 98 & 4.09 & 9 & 119 & & 5.4 & 2 & 95 & 110 \\
\hline 5 & 31 & 77 & 1.76 & 1.92 & 3.92 & 93 & 4.79 & 91 & 82 & 100 & 5.92 & 8 & 9 & & - & & 94 & 79 \\
\hline Mean & 33 & 61 & 1.65 & 1.63 & 3.22 & 92 & 4.11 & 98 & 79 & 96 & 5.35 & 93 & 91 & 12.6 & 5.0 & 7.43 & 94 & 104 \\
\hline SD & ? & 12 & 0.1 & 0.21 & 0.70 & 5 & 1.00 & 7 & 5 & 5 & 1.20 & 6 & 20 & 1.0 & 06 & 0.02 & 1 & 20 \\
\hline \multicolumn{19}{|l|}{ Children } \\
\hline 6 & 10 & 3 & 1.36 & & 1.60 & 91 & 2.0 & 96 & 78 & 92 & 3.1 & 11 & 10 & & & & 95 & 152 \\
\hline 7 & 16 & 40 & 1.49 & 1. & 1.80 & 75 & 2.61 & 90 & 70 & 83 & 3.7 & 99 & 12 & 13 & 5 & 5 & 96 & 96 \\
\hline 8 & 14 & 3 & 1.41 & 1.17 & 1.99 & 98 & 2.64 & 106 & 83 & 98 & 2.95 & 92 & 107 & 14.8 & 4.4 & 7.46 & 96 & 116 \\
\hline 9 & 7 & 23 & 1.21 & 0.88 & 1.38 & 105 & 1.61 & 99 & 84 & 98 & 2.28 & 106 & - & 14.3 & 4.8 & 7.44 & 96 & 113 \\
\hline 10 & 6 & 21 & 1.17 & 0.83 & 1.19 & 103 & 1.49 & 104 & 88 & 102 & 1.67 & 87 & - & - & - & - & 97 & - \\
\hline Mean & 12 & 36 & 1.37 & 1.05 & 1.59 & 94 & 2.08 & 99 & 81 & 85 & 2.74 & 99 & 110 & 13.8 & 5.1 & 7.44 & 96 & 119 \\
\hline SD & 5 & 16 & 0.15 & 0.19 & 0.32 & 12 & 0.54 & 6 & 7 & 0.8 & 0.79 & 10 & 9 & 0.9 & 0.6 & 0.02 & 1 & 24 \\
\hline
\end{tabular}

BSA: body surface area; FEV1: forced expiratory volume in one second; VC: vital capacity; TLC: total lung capacity; KCO: carbon monoxide transfer coefficient; $\mathrm{Pa}, \mathrm{O}_{2}$ and $\mathrm{Pa}_{\mathrm{a}} \mathrm{CO}_{2}$ : arterial oxygen and carbon dioxide tension, respectively; $\mathrm{Sa}, \mathrm{O}_{2}$ : arterial oxygen saturation; $\mathrm{Hb}$ : haemoglobin; \% pred: percentage of predicted value. 
Table 2. - Exercise capacity data

\begin{tabular}{|c|c|c|c|c|c|c|c|c|c|c|c|c|c|c|c|c|c|}
\hline \multirow[t]{2}{*}{$\begin{array}{l}\text { Patients } \\
\text { no. }\end{array}$} & \multirow[t]{2}{*}{ Protocol } & \multirow{2}{*}{$\begin{array}{c}\text { Dura- } \\
\text { tion } \\
\text { min }\end{array}$} & \multicolumn{2}{|c|}{ Peak $V^{\prime} \mathrm{O}_{2}$} & \multirow{2}{*}{$\begin{array}{c}\text { Max } \\
\text { power } \\
\text { W }\end{array}$} & \multicolumn{2}{|c|}{$\operatorname{Max} f \mathrm{C}$} & \multirow[t]{2}{*}{$\begin{array}{c}\text { End ex. } \\
\text { RER }\end{array}$} & \multicolumn{2}{|c|}{$\begin{array}{c}\text { End exercise } \\
V^{\prime} \mathrm{E}\end{array}$} & \multirow{2}{*}{$\begin{array}{c}\text { Rest } \\
\mathrm{Sa}_{\mathrm{a}, \mathrm{O}_{2}} \\
\%\end{array}$} & \multirow{2}{*}{$\begin{array}{c}\text { End ex. } \\
\mathrm{S}_{\mathrm{a}, \mathrm{O}_{2}} \\
\%\end{array}$} & \multirow{2}{*}{$\begin{array}{l}\text { Lactat- } \\
\text { aemia } \\
\mathrm{mmol} \cdot \mathrm{L}^{-1}\end{array}$} & \multirow{2}{*}{$\begin{array}{c}\mathrm{VT} \\
\% \text { pred } \\
\mathrm{p} V^{\prime} \mathrm{O}_{2}\end{array}$} & \multirow{2}{*}{$\begin{array}{c}V^{\prime} \mathrm{E} / \\
V^{\prime} \mathrm{O}_{2} \\
\text { at VT }\end{array}$} & \multirow{2}{*}{$\begin{array}{c}V^{\prime} \mathrm{E} / \\
V^{\prime} \mathrm{CO}_{2} \\
\text { at VT }\end{array}$} & \multirow{2}{*}{$\begin{array}{c}V^{\prime} \mathrm{O}_{2} / f \mathrm{C} \\
\text { slope } \\
\mathrm{mL} \cdot \mathrm{kg}^{-1} . \\
\mathrm{min}^{-2}\end{array}$} \\
\hline & & & $\begin{array}{c}\mathrm{mL} \cdot \mathrm{kg}^{-1} \\
\min ^{-1}\end{array}$ & $\begin{array}{c}\% \\
\text { pred }\end{array}$ & & $\begin{array}{l}\text { beats. } \\
\text { min }^{-1}\end{array}$ & $\begin{array}{c}\% \\
\text { pred }\end{array}$ & & $\mathrm{L} \cdot \min ^{-1}$ & $\begin{array}{c}\% \\
\text { MVV }\end{array}$ & & & & & & & \\
\hline \multicolumn{18}{|l|}{ Adults } \\
\hline 1 & Cycle & 12.0 & 29.7 & 77 & 135 & 155 & 82 & 1.20 & 77.5 & 54 & 98 & 96 & 7.1 & 44 & 20.3 & 31.0 & 0.19 \\
\hline 2 & Cycle & 10.5 & 28.2 & 87 & 120 & 180 & 97 & 1.26 & 54.0 & 58 & 97 & 97 & 9.0 & 58 & 22.7 & 28.0 & 0.22 \\
\hline 3 & Cycle & 9.0 & 24.9 & 64 & 105 & 190 & 101 & 1.23 & 49.3 & 45 & 97 & 96 & 8.1 & 38 & 24.5 & 26.0 & 0.34 \\
\hline 4 & Cycle & 9.0 & 21.9 & 67 & 75 & 174 & 92 & 1.10 & 36.5 & 45 & 98 & 96 & 5.2 & 52 & 24.2 & 34.4 & 0.21 \\
\hline 5 & Cycle & 11.0 & 34.0 & 85 & 165 & 169 & 88 & 1.19 & 87.0 & 64 & 97 & 95 & 6.3 & 47 & 24.6 & 27.3 & 0.29 \\
\hline Mean & & 10.3 & 27.7 & 76 & 120 & 174 & 92 & 1.20 & 60.9 & 53 & 97 & 96 & 7.1 & 48 & 23.3 & 29.3 & 0.25 \\
\hline $\mathrm{SD}$ & & 1.3 & 4.6 & 10 & 34 & 13 & 7 & 0.06 & 20.8 & 8 & 1 & 1 & 1.5 & 8 & 1.8 & 3.4 & 0.06 \\
\hline \multicolumn{18}{|l|}{ Children } \\
\hline 6 & Cycle & 9.0 & 44.0 & 121 & 135 & 190 & 90 & 1.07 & 41.0 & 73 & 98 & 95 & 5.9 & 73 & 19.8 & 26.0 & 0.44 \\
\hline 7 & Cycle & 10.0 & 43.0 & 97 & 123 & 190 & 93 & 1.10 & 60.0 & 71 & 99 & 97 & 6.8 & 67 & 24.0 & 31.2 & 0.39 \\
\hline 8 & Cycle & 8.5 & 42.8 & 99 & 110 & 188 & 92 & 1.01 & 46.4 & 66 & 99 & 96 & 6.2 & 55 & 21.8 & 30.0 & 0.48 \\
\hline 9 & Treadmill & 9.3 & 43.0 & 109 & - & 176 & 83 & 0.90 & 31.4 & 68 & 96 & 96 & - & 70 & 29.0 & 38.0 & 0.53 \\
\hline 10 & Treadmill & 8.5 & 32.6 & 78 & - & 180 & 88 & 1.06 & 20.1 & 48 & - & - & - & - & - & - & 0.28 \\
\hline Mean & & 9.1 & 41.1 & 101 & 123 & 185 & 89 & 1.03 & 39.8 & 65 & 98 & 96 & 6.3 & 66 & 23.7 & 31.3 & 0.42 \\
\hline SD & & 0.6 & 4.7 & 16 & 13 & 6 & 4 & 0.08 & 15.1 & 10 & 1 & 1 & 0.5 & 8 & 4.0 & 5.0 & 0.10 \\
\hline
\end{tabular}

$V^{\prime} \mathrm{O}_{2}$ : oxygen consumption; $f \mathrm{C}$ : cardiac frequency; ex.: exercise; RER: respiratory exchange ratio; $V^{\prime} \mathrm{E}$ : ventilation; $S_{\mathrm{a}, \mathrm{O}_{2}}$ : arterial oxygen saturation; VT: ventilatory threshold; $\mathrm{p} V^{\prime} \mathrm{O}_{2}$ : peak $V^{\prime} \mathrm{O}_{2} ; V^{\prime} \mathrm{E} / V^{\prime} \mathrm{O}_{2}$ and $V^{\prime} \mathrm{E} / V^{\prime} \mathrm{CO}_{2}$ : ventilatory equivalents for oxygen and carbon dioxide, respectively; $\%$ pred: percentage of predicted value; MVV: maximal voluntary ventilation.

relation coefficient $r$ reached $0.93-0.99)$. Adult patients had a significantly lower slope of the $V^{\prime} \mathrm{O}_{2} / f \mathrm{C}$ relationship (i.e. a lower increase in $V^{\prime} \mathrm{O}_{2}$ associated with a given increase in $f \mathrm{C}$ ) than children (mean slope $=0.25 \pm 0.06$ versus $\left.0.42 \pm 0.10 \mathrm{~mL} \cdot \mathrm{kg}^{-1} \cdot \mathrm{min}^{-2}, \mathrm{p}=0.02\right)$. The mean values of the $V^{\prime} \mathrm{O}_{2} / f \mathrm{C}$ slope normalized for body weight in 107 children ranging 6-17 yrs described by COOPER et al. [17] were $0.37 \pm 0.10 \mathrm{~mL} \cdot \mathrm{kg}^{-1} \cdot \mathrm{min}^{-2}$ for the male children and $0.29 \pm$ $0.08 \mathrm{~mL} \cdot \mathrm{kg}^{-1} \cdot \mathrm{min}^{-2}$ for the female children.

\section{Echocardiography}

Echocardiographic measurements are shown in table 3. The estimated cardiac index was elevated (group mean at $3.9 \pm 0.8 \mathrm{~L} \cdot \mathrm{min}^{-1} \cdot \mathrm{m}^{-2}$, compared with a normal value of $2.9 \pm$ $\left.0.4 \mathrm{~L} \cdot \mathrm{min}^{-1} \cdot \mathrm{m}^{-2}\right)$. Left systolic dysfunction was present in one patient (patient No. 4) with a shortening fraction of the left ventricle of $27 \%(\mathrm{~N}=36 \pm 4 \%)$. Group mean short- ening fraction, however, was normal: $39 \pm 6 \%$ and three patients had a shortening fraction $>40 \%$ (patients No. 3, 9 and 10). Moderate LV end-diastolic dilatation was present in three adult patients (patients No. 1, 3 and 4). LVPW thickness was normal in all subjects and IVS thickness was moderately increased for patients No. 1 and 5. sPAP was significantly higher in adults $(33 \pm 4 \mathrm{mmHg}$, range $28-$ $37 \mathrm{mmHg})$ than in children $(24 \pm 3 \mathrm{mmHg}$, range $20-28$ $\mathrm{mmHg} ; \mathrm{p}=0.01$ ). Diastolic dimensions of the right ventricle were higher than normal in both adults and children.

\section{Correlations}

Significant correlations were found between peak $V^{\prime} \mathrm{O}_{2}$ (\% pred) and the slope of $V^{\prime} \mathrm{O}_{2} / f \mathrm{C}(\mathrm{p}=0.01)$, with the peak $V^{\prime} \mathrm{O}_{2}$ being maximally lowered in those patients with the lowest slopes. Peak $V^{\prime} \mathrm{O}_{2}$ tended to be otherwise inversely correlated with age $(\mathrm{p}=0.08)$ and with the duration for
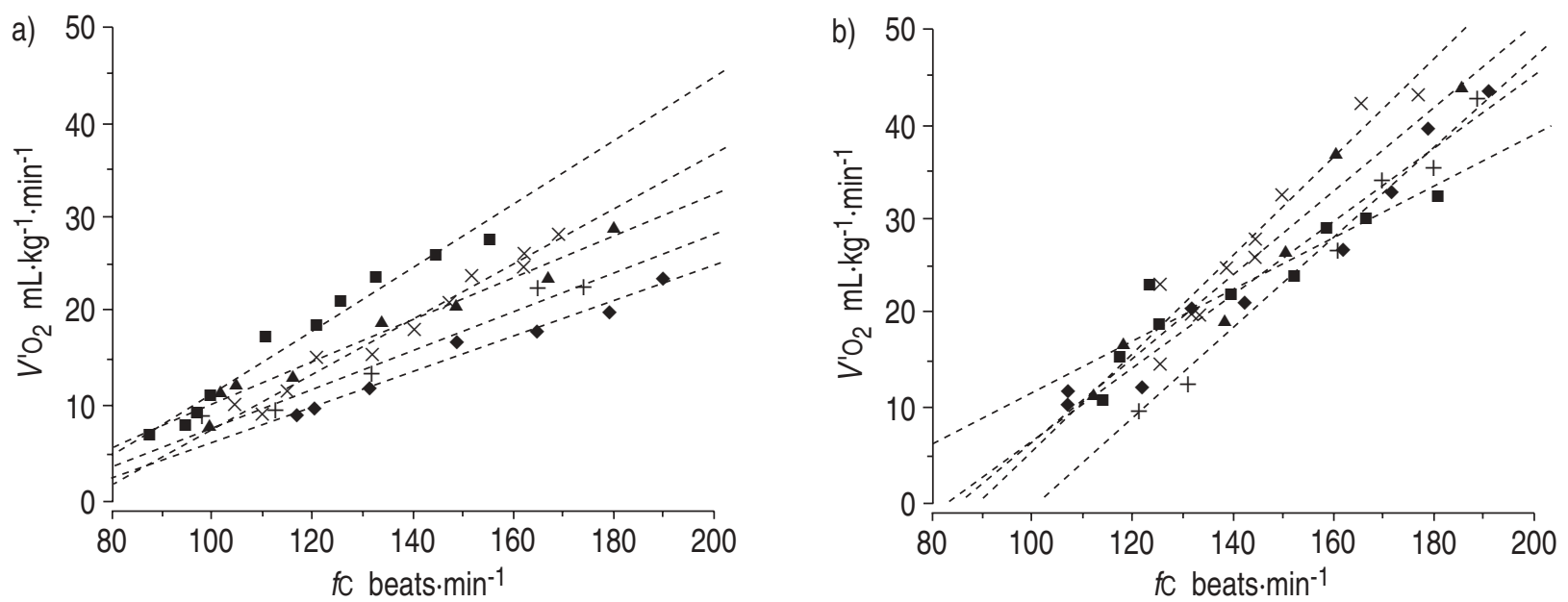

Fig. 1. - Relationship between oxygen consumption $\left(V^{\prime} \mathrm{O}_{2}\right)$ and cardiac frequency $(f \mathrm{C})$ for a) the five adults and b) the five children. a) $\mathbf{a}$ : subject no. 1; $\boldsymbol{\Delta}: 2 ; \diamond: 3 ;+: 4 ; \times: 5 ;$ b) $\mathbf{\Delta}:$ subject no. $6 ; \diamond: 7 ;+: 8 ; \times: 9 ;$ a: 10 . Mean correlation coefficient=0.975 (range 0.93-0.99). 
Table 3. - Echocardiographic data

\begin{tabular}{|c|c|c|c|c|c|c|c|c|}
\hline $\begin{array}{l}\text { Patients } \\
\text { No. }\end{array}$ & $\begin{array}{l}\text { LV diastolic } \\
\text { diameter } \\
\mathrm{cm} \cdot \mathrm{m}^{-2}\end{array}$ & $\begin{array}{l}\text { RV diastolic } \\
\text { diameter } \\
\mathrm{cm} \cdot \mathrm{m}^{-2}\end{array}$ & $\begin{array}{l}\text { IVS } \\
\mathrm{cm}\end{array}$ & $\begin{array}{c}\text { LVPW } \\
\mathrm{cm}\end{array}$ & $\begin{array}{c}\text { LV } \\
\text { Shorten fract. } \\
\%\end{array}$ & $\begin{array}{c}\mathrm{RV} \\
\text { Shorten fract. } \\
\%\end{array}$ & $\begin{array}{l}\text { Cardiac index } \\
\mathrm{L} \cdot \mathrm{min}^{-1} \cdot \mathrm{m}^{-2}\end{array}$ & $\begin{array}{l}\text { sPAP } \\
\text { mmHg }\end{array}$ \\
\hline \multicolumn{9}{|l|}{ Adults } \\
\hline 1 & 3.4 & 1.5 & 1.3 & 0.8 & 40 & 52 & 5.4 & 35 \\
\hline 2 & 3.0 & 2.0 & 0.9 & 0.8 & 38 & 37 & 3.0 & 28 \\
\hline 3 & 3.4 & 1.6 & 1.0 & 0.9 & 44 & 49 & 4.3 & 37 \\
\hline 4 & 3.9 & 2.2 & 0.9 & 0.8 & 27 & 32 & 3.7 & 30 \\
\hline 5 & 3.2 & 1.5 & 1.2 & 1.0 & 39 & 31 & 5.1 & 36 \\
\hline Mean & 3.4 & 1.7 & 1.1 & 0.9 & 38 & 40 & 4.3 & 33 \\
\hline SD & 0.3 & 0.3 & 0.2 & 0.1 & 6 & 10 & 1.0 & 4 \\
\hline Normal & $2.1-3.2$ & $0.4-1.4$ & $0.6-1.1$ & $0.6-1.1$ & $36 \pm 4$ & $40 \pm 3$ & $2.9 \pm 4$ & $<35$ \\
\hline \multicolumn{9}{|l|}{ Children } \\
\hline 6 & $4.2(3.0-4.3)$ & $2.2(0.6-1.6)$ & $0.7(0.6-0.8)$ & $0.6(0.6-0.8)$ & 33 & 38 & 2.8 & 28 \\
\hline 7 & $3.0(2.6-3.6)$ & $1.1(0.6-1.3)$ & $0.7(0.6-0.8)$ & $0.7(0.6-0.8)$ & 35 & 44 & 3.3 & 25 \\
\hline 8 & $3.8(2.8-4.0)$ & $1.9(0.7-1.4)$ & $0.6(0.6-0.8)$ & $0.6(0.6-0.8)$ & 40 & 45 & 4.2 & 20 \\
\hline 9 & $4.6(2.7-4.8)$ & $2.1(0.5-2.0)$ & $0.5(0.5-0.7)$ & $0.4(0.5-0.7)$ & 49 & 37 & 4.0 & 22 \\
\hline 10 & $4.3(2.9-5.0)$ & $2.6(0.5-2.1)$ & $0.6(0.5-0.7)$ & $0.6(0.5-0.7)$ & 42 & 41 & 4.6 & 25 \\
\hline Mean & 4.0 & 2.0 & 0.6 & 0.6 & 40 & 41 & 3.8 & 24 \\
\hline SD & 0.6 & 0.6 & 0.1 & 0.1 & 6 & 4 & 0.7 & 3 \\
\hline
\end{tabular}

Ranges are shown in parentheses. LV: left ventricle; RV: right ventricle; IVS: interventricular septum thickness; LVPW: LV posterior wall thickness; Shorten fract: shortening of ventricular diameter during systole; sPAP: systolic pulmonary arterial pressure.

which serum ferritin was elevated $(\mathrm{p}=0.05)$. No correlation was found between peak $V^{\prime} \mathrm{O}_{2}$ and $\mathrm{Hb}$ level $(\mathrm{p}=0.10)$ or shortening fraction of the left ventricle measured at rest $(\mathrm{p}=0.77)$. A significant inverse correlation was found between sPAP and $K \mathrm{CO}(\mathrm{p}=0.05)$. sPAP correlated with age $(p=0.03)$ and with the duration for which the ferritin level was elevated $(\mathrm{p}=0.02)$.

\section{Discussion}

The main finding of this study was a significant impairment in exercise capacity in thalassaemic adult patients, which was not observed in children. No evidence was found in favour of a pulmonary parenchymal or vascular involvement responsible for exercise limitation. The abnormal cardiac response may have contributed to the exercise limitation observed.

The decrease in $\mathrm{KCO}$ and bronchial obstruction in two patients, both of which were of mild intensity, were the only respiratory abnormalities observed in this population. Although the nature of the ventilatory defect (i.e. restrictive versus obstructive) in thalassaemia is not entirely clear, bronchial obstruction has occasionally been reported and this was associated with hyperinflation and hypoxaemia; small airway disease due to iron deposition may have been a causative factor [4]. Recent reports on lung function immediately after transfusion have shown that thalassaemic patients with a clinical history of airway obstruction and a decrease in FEV1 and forced expiratory flow between 25 and $75 \%$ vital capacity (FEF25-75\%) had a worsening of their airway obstruction after transfusion [5]. This suggests that airway narrowing may, at least in part, be due to airway wall vascular engorgement and/or oedema induced acutely and aggravated by transfusion. Cardiac dysfunction may potentially enhance this phenomenon.

TL,CO was also minimally affected in this group of patients. It should be noted, however, that this occurred in adult patients and not in the three children studied where, conversely, KCO tended to be above the normal range. A similar increase in $\mathrm{KCO}$ was observed in other studies of thalassaemic children [3]. Factors responsible for this increase have been attributed either to structural changes in relation to the disease (slowing in the growth of the airspace relative to the vascular bed) or to functional changes related to the increase in pulmonary perfusion. Conversely, a decrease in $\mathrm{KCO}$ has been reported in thalassaemic patients, including adult patients [8]. Careful determination of a gas transfer factor of the pulmonary membrane suggested that this decrease could primarily be due to an alveolocapillary membrane block. This was additionally associated with a decrease in TLC and exercise desaturation. Infiltration by abnormal erythroid cells (ineffective erythropoiesis) has been proposed to explain this impairment [11]. An alternative explanation for the decrease in KCO may be a consequence of pulmonary artery disease observed by several investigators in thalassaemia. In a study by GRISARU et al. [7] on 35 patients with homozy-gous $\beta$-thalassaemia, pulmonary hypertension was detect-ed in $74 \%$ of patients by echocardiography. In a different population [11], high levels of sPAP $(55-90 \mathrm{mmHg})$ have been reported using right heart catheterization. However, pulmonary artery disease was not thought to be present in the present patients. Exercise testing is considered a sensitive tool for detecting pulmonary vascular disease. Neither a significant increase in dead space ventilation (as re-flected by a normal $V^{\prime} \mathrm{E} / V^{\prime} \mathrm{CO}_{2}$ at ventilatory threshold) nor arterial oxygen desaturation during exercise (a promin- ent feature of pulmonary vascular disease) was observed in these patients. The exact behaviour of KCO during the course of thalassaemia is unknown; in particular, the possibility that an increase in $\mathrm{KCO}$ (as observed in children) may precede a subsequent decrease (as observed in adulthood) would require further studies.

A significant decrease in exercise capacity was seen in adult thalassaemic patients (mean age 33 yrs) which was not observed in the subgroup of children (mean age 12 yrs). The main differences between these two groups were the duration of evolution of the disease and the fact that 
splenectomy was performed only in adults. No arguments were found in favour of a ventilatory limitation in this group of patients. A relative hypoventilation during exercise in thalassaemia has been hypothesized by GRANT et al. [17]. These authors observed a decrease in the $V^{\prime} \mathrm{E} / \mathrm{V}$ ' $\mathrm{CO}_{2}$ ratio associated with an increase in "estimated" $\mathrm{PCO}_{2}$. Similar findings have been reported by Ross et al. [18]. In the present study, mean $V^{\prime} \mathrm{E} / V^{\prime} \mathrm{CO}_{2}$ at the ventilatory threshold were of the same range as those noted for control subjects at $45 \%$ of maximal power in the study of GRANT et al. [17]. The reasons for these differences are unclear. There are several arguments in favour of a cardiac limitation in this group of adult patients: 1) none of them showed ventilatory limitation; 2 ) all reached a near-maximal $f C$ at the end of exercise; and 3) a decrease in ventilatory threshold was observed in the most affected patient (patient No. 3) and the ventilatory threshold tended to be lower in affected adult patients than in children. The slope of the $V^{\prime} \mathrm{O}_{2} / f \mathrm{C}$ relationship is an additional indicator of systolic ejection function during exercise $[15,19]$. A low slope of $V^{\prime} \mathrm{O}_{2} / f \mathrm{C}$ indicates that a high $f \mathrm{C}$ increase is associated with a given increase in $V^{\prime} \mathrm{O}_{2}$. A reduced stroke volume during exercise is associated with a steep $f \mathrm{C}$ response (i.e. a low slope of $V^{\prime} \mathrm{O}_{2} / f \mathrm{C}$ ). COOPER et al. [19] extensively studied such relationships in children and showed that the value of the slope during exercise was principally affected by weight (presumably reflecting muscular mass). Thus, the slope of $V^{\prime} \mathrm{O}_{2} / f \mathrm{C}$ normalized for differences in weight is independent of age [19]. In the present study, all values of the $V^{\prime} \mathrm{O}_{2} / f \mathrm{C}$ slope were within normal limits (mean \pm $2 \mathrm{sD}$ ), or elevated in children. Conversely, adults had significantly lower values of the slope of $V^{\prime} \mathrm{O}_{2} / f \mathrm{C}$ than children and the slope value correlated negatively and maximally with the time spent with a high serum ferritin level $(\mathrm{p}=$ 0.04). Other studies have documented an impairment in exercise capacity in thalassaemia. In a group of 13 thalassaemic patients $12-22$ yrs old, the mean $V^{\prime} \mathrm{O}_{2}$ achieved was $28.7 \pm 2.1$ versus $38.0 \pm 7.9 \mathrm{~mL} \cdot \mathrm{kg}^{-1} \cdot \mathrm{min}^{-1}$ in controls [17]. Similar $f C$ were reached at the end of exercise in both groups, and cardiac output at a given $V^{\prime} \mathrm{O}_{2}$ was higher in thalassaemic patients. Surprisingly, none of these parameters was influenced by transfusion.

Anaemia is a possible confounding factor which may have affected peak $V^{\prime} \mathrm{O}_{2}[20]$ as well as the haemodynamic response to submaximal exercise [21]. The degree of anaemia is important to consider, however, and moderate levels of anaemia (two-thirds or more of normal $\mathrm{Hb}$ level) are associated with limited cardiovascular consequences and preserved $\mathrm{O}_{2}$ transport [22]. It should be emphasized that the present patients were generally moderately anaemic (mean $\mathrm{Hb} 112 \mathrm{~g} \cdot \mathrm{L}^{-1}$ ). The precise effects of anaemia on the $V^{\prime} \mathrm{O}_{2} / f \mathrm{C}$ relationships are conflicting. In a study by MLLER $e t$ al. [21], the authors suggested that the cardiac response associated with a given $V^{\prime} \mathrm{O}_{2}$ is affected more by the amount of muscular mass than by the degree of anaemia. Thus changes in $V^{\prime} \mathrm{O}_{2}$ normalized for body weight, as in the present study should be less affected. However, COOPER et al. [23] provided direct evidence that the level of $\mathrm{Hb}$ (modified by transfusions) may at least in part influence the $V^{\prime} \mathrm{O}_{2} / f \mathrm{C}$ relationship. This effect, however, was of limited importance in the subgroup of older patients, with a $13 \%$ increase in the $V^{\prime} \mathrm{O}_{2} / f \mathrm{C}$ slope after transfusion in the group of patients $>12$ yrs old [23]. It is unlikely that differences in the $V^{\prime} \mathrm{O}_{2} / f \mathrm{C}$ slope between children and adults in the present study can be explained by differences in the level of $\mathrm{Hb}$ which was, additionally, not significantly different between children and adults in this population.

A normal or increased slope, as observed in the group of children, would suggest a normal cardiac response to exercise in this group. Conversely, the decrease in the slope of $V^{\prime} \mathrm{O}_{2} / f \mathrm{C}$ observed in adults may reflect an impairment of cardiac function. The significance of this observation could be a lower stroke volume on exercise in adults than in children. LV dysfunction during exercise has been proposed as an early marker of cardiac impairment in thalassaemia [24], followed by moderate ventricular dilatation and LV dysfunction at rest later in the course of the disease $[7,25]$. Other authors have described an abnormal contraction and relaxation of the posterior LV wall [26]. Such abnormalities may precede concentric LV thickening, which occurred in $75 \%$ of thalassaemic patients in the study by HeNRY et al. [27]. None of these abnormalities, however, was seen in the present study. Echocardiographic measurements in this study showed an elevated cardiac index in both adults and children, which inversely correlated with $\mathrm{Hb}$ level. This may have artificially enhanced the resting shortening fraction and precluded the sensitive evaluation of cardiac impairment.

In conclusion, an abnormal cardiac response on exercise was the most frequent abnormality observed in this group of adult patients with limited pulmonary involvement. The early detection of cardiac abnormalities detection may have important therapeutic and prognostic implications in thalassaemia as well as other causes of secondary haemosiderosis. Further studies would be warranted to establish the long-term reproducibility of indices derived from exercise testing as a tool to detect early cardiac dysfunction in the course of this disease.

Acknowledgements: The authors wish to thank I. Pin and J-P. Gout for helpful discussions and positive support at the initiation of this work. They also acknowledge the contributions of $\mathrm{M}$. Bost and $\mathrm{C}$. Bachelot for referring the patients and A.M. Rossignol and O. Hadjian for performing cardiac echographies.

\section{References}

1. Zurlo MG, De Stephano P, Borgna-Pignatti C. Survival and causes of death in major thalassemia. Lancet 1989; ii: 27-30.

2. Olivieri NF, Nathan DG, MacMillan JH, et al. Survival in medically treated patients with homozygous $\beta$-thalassemia. N Engl J Med 1994; 331: 574-578.

3. Cooper DM, Mansell AL, Weiner MA, et al. Low lung capacity and hypoxemia in children with thalassemia major. Am Rev Respir Dis 1980; 121: 639-646.

4. Keens TG, O'Neal MH, Ortega JA, Hyman CB, Platzker ACG. Pulmonary function abnormalities in thalassemia patients on a hypertransfusion program. Pediatrics 1980; 65: 1013-1017.

5. Santamaria F, Villa MP, Werner B, Cutrera R, Barreto M, Ronchetti R. The effect of transfusion on pulmonary function in patients with thalassemia major. Pediatr Pulmonol 1994; 18: 139-143.

6. Factor JM, Pottipati SR, Rappaport I, Rosner IK, Lesser ML, Giardrna PJ. Pulmonary function abnormalities in thalassemia major and the role of iron overload. Am J 
Respir Crit Care Med 1994; 149: 1570-1574.

7. Grisaru D, Rachmilewitz EA, Mosseri M, et al. Cardiopulmonary assessment in $\beta$-thalassemia major. Chest 1990; 98: 1138-1142.

8. Thai DYA, Wang YT, Lou J, Malk KH, Cheng HK. Lungs in thalassemia major patients receiving regular transfusion. Eur Respir J 1996; 9: 1389-1394.

9. Grant GP, Mansell AL, Graziano JH, Mallins RB. The effect of transfusion on lung capacity, diffusing capacity, and arterial oxygen saturation in patients with thalassemia major. Pediatr Res 1986; 20: 20-23.

10. Sonakul D, Pacharee P, Laohapand T, Fucharoen S, Wasi P. Pulmonary artery obstruction in thalassemia. SE Asian J Trop Med Public Health 1980; 11: 516-523.

11. Aessopos A, Stamatelos G, Skoumas V, Vassilopoulos G, Mantzourani M, Loukopoulos D. Pulmonary hypertension and right heart failure in patients with $\beta$-thalassemia intermedia. Chest 1995; 107: 50-53.

12. Quanjer PH. Standardized lung function testing. Report working party "standardization of lung function tests", European Community for Coal and Steel. Bull Eur Physiopathol Respir 1983; 19: Suppl. 5, 1-95.

13. Zapletal A, Samanek M, Paul T. Lung function in children and adolescents. In: Herzog H, eds. Progress in Respiratory Research, Vol. 17. Basel, Karger, 1981; pp. 52-67.

14. Cotes JE, Chinn DJ, Quanjer PhH, Roca J, Yernault JC. Standardisation of the measurement of the transfer factor (diffusing capacity). Eur Respir J 1993; 6: Suppl. 16, 41-52.

15. Wasserman K, Hansen JE, Sue DY, Whipp BJ. Principles of Exercise Testing and Interpretation. Chapter 6. Normal values. Philadelphia, PA, Lea \& Febiger, 1987.

16. Feigenbaum H, eds. Echocardiography, 4th Edn. Philadelphia, PA, Lea and Febiger, 1986.

17. Grant GP, Seaman GC, Mansell AL. Cardiorespiratory response to exercise in patients with thalassemia major. Am Rev Respir Dis 1987; 136: 92-97.

18. Ross RD, Wessel HU, Paul MH. Reduced ventilatory response to graded exercise in patients with thalassemia major. Fed Proc 1984; 43: 634.

19. Cooper DM, Weiler-Ravell D, Whipp BJ, Wasserman K. Growth-related changes in oxygen uptake and heart rate during progressive exercise in children. Pediatr Res 1984; 18: 845-851.

20. Sproule BJ, Mitchell JH, Miller WF. Cardiopulmonary physiological response to heavy exercise in patients with anemia. J Clin Invest 1960; 39: 378-388.

21. Miller GJ, Serjeant GR, Sivapragasam S, Petch MC. Cardio-pulmonary response and gas exchange during exercise in adults with homozygous sickle-cell disease (sickle-cell anaemia). Clin Sci 1973; 44: 113-128.

22. Finch CA, Lenfant C. Oxygen transport in man. $N$ Engl $J$ Med 1972; 286: 407-415.

23. Cooper DM, Hyman CB, Weiler-Ravel D, Noble NA, Agness CL, Wasserman K. Gas exchange during exercise in children with thalassaemia major and Diamond-Blackfan anemia. Pediatr Res 1985; 19: 1215-1219.

24. Freeman AP, Giles RW, Berdoukas VA, Walsh WF, Choy D, Murray PC. Early left ventricle dysfunction and chelation therapy in thalassemia major. Ann Intern Med 1983; 99: 450-454.

25. Krestaminos DT, Tontouzos PK, Vyssoulis GP, Vinitis CA, Vretan HF, Avgonstakis DG. Global and segmental left ventricular function in $\beta$-thalassemia. Cardiology 1985; 72: 129-139.

26. Valdes-Cruz LM, Reineeke C, Rutkowski M, et al. Preclinical abnormal segmental cardiac manifestations of thalassemia major in children on transfusion-chelation therapy: echographic alterations of left ventricular posterior wall contraction and relaxation patterns. Am Heart $J$ 1982; 103: 505-510.

27. Henry WL, Nienhuis AW, Weiner M, Miller DR, Canale VC, Piomelli S. Echocardiographic abnormalities in patients with transfusion-dependent anemia and secondary myocardial iron deposition. Am J Med 1978; 64: 547555. 